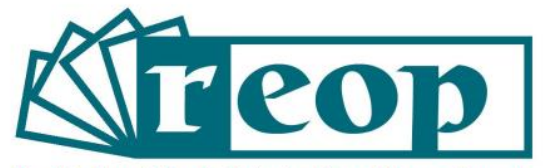

Revista Española de Orientación y Psicopedagogía

\title{
PROGRAMA DE ORIENTACIÓN ENTRE IGUALES EN EDUCACIÓN SUPERIOR PARA LA ADQUISICIÓN DE COMPETENCIAS INSTRUMENTALES ${ }^{1}$
}

\section{A PEER GUIDANCE PROGRAM IN HIGHER EDUCATION FOR THE ACQUISITION OF INSTRUMENTAL SKILLS}

\author{
Noemí Merayo Álvarez ${ }^{2}$ \\ Universidad de Valladolid. Escuela Técnica Superior de Ingenieros de Telecomunicación. \\ Departamento de Teoría de la Señal, Comunicación e ingeniería telemática. Valladolid, España \\ Inés Ruiz-Requies ${ }^{3}$ \\ Universidad de Valladolid. Facultad de Educación y Trabajo Social. Departamento de Pedagogía. \\ Valladolid, España \\ Nicole Ávalos Díaz ${ }^{4}$ \\ Universidad Católica Silvia Henríquez. Santiago de Chile, Chile
}

\section{RESUMEN}

Las necesidades detectadas en los estudiantes de nuevo ingreso de las Escuelas Técnicas Superiores de Ingenieros de Telecomunicación han llevado a plantear iniciativas de mentoría formal en sus grados. Estas carencias están relacionadas con una escasa orientación previa a la

\footnotetext{
1 Fuente de financiación: Este trabajo ha sido financiado por la Universidad de Valladolid como Proyecto de Innovación Educativa MENTUm durante los años 2015, 2016, 2017 y 2018. 1 Premio del Consejo Social de la Universidad de Valladolid, Edición 2016 "MENTUm: Diseño de un Sistema de Mentorización Integral Profesores-Alumnos Mentores en la E.T.S.I. Telecomunicación de la Universidad de Valladolid".

2 Correspondencia: Noemí Merayo Álvarez. Correo-e: noemer@tel.uva.es
} 
Universidad, gran heterogeneidad del alumnado, alto índice de fracaso académico en el primer curso y falta de información acerca de la inserción laboral. Este artículo pretende dar a conocer el programa MENTUm cuya finalidad es que estudiantes de últimos cursos ofrezcan apoyo e incentiven el aprendizaje e integración de estudiantes de primero con el objetivo de detectar las dificultades personales y académicas que presentan y desarrollar competencias instrumentales. La metodología de investigación empleada consiste en un estudio de caso desde un enfoque mixto, empleando técnicas tanto cuantitativas como cualitativas, con la finalidad de alcanzar la mayor comprensión del programa en una de sus cuatro dimensiones: dar respuesta a las necesidades del alumnado de nuevo ingreso. Para la recogida de información, se han empleado tres técnicas de recogida de datos: cuestionarios, observaciones y entrevistas. Los resultados muestran que las dificultades personales tienen que ver con la falta de concentración, planificación y organización del tiempo, mientras que las dificultades académicas tienen que ver más con la falta de conocimientos previos ante los contenidos de las asignaturas. Como conclusiones hemos constatado que los estudiantes son conscientes desde su ingreso a la Universidad de la importancia de desarrollar competencias instrumentales de aprendizaje autónomo y planificación a medida que transcurre el año académico.

Palabras clave: Competencias instrumentales, coaching, mentores, mentorizados, orientación.

\section{ABSTRACT}

The needs detected in the new students at the Higher Technical Schools of Telecommunication Engineers have led to propose initiatives of formal mentoring in their grades. These shortcomings are related to a poor orientation prior to the University, great heterogeneity of the students, high rate of academic failure in the first year and lack of information about the labor insertion. This article describes the MENTUm program, whose purpose is for senior students to support and encourage the learning and integration of first-year students with the aim of detecting the personal and academic difficulties they present and developing some instrumental skills. The research methodology used consists of a case study from a mixed approach, using both quantitative and qualitative techniques to achieve a greater understanding of the program in one of its four dimensions: respond to the needs of new students. For the collection of information, three data collection techniques have been used: questionnaires, observations, and interviews. The results show that personal difficulties have to do with the lack of concentration, planning and organization of time, while academic difficulties have more to do with the lack of prior knowledge regarding the contents of the subjects. As conclusions, we have verified that the students are aware, from the beginning, upon their entrance into the University, of the importance of developing instrumental skills of autonomous learning and planning as the academic year progresses.

Key Words: Instrumental skills coaching, mentors, mentorized, Guidance.

\section{Cómo citar este artículo:}

Merayo, N. Ruiz-Requies, I. y Ávalos, N. (2021). Programa de orientación entre iguales en Educación Superior para la adquisición de competencias instrumentales. Revista Española de $\begin{array}{llll}\text { Orientación } & y & \text { Psicopedagogía, } & 32(1),\end{array}$ https://doi.org/10.5944/reop.vol.32.num.1.2021.30745 


\section{Introducción}

La orientación universitaria es un proceso continuo de acompañamiento al estudiante en su transición desde el Bachillerato o la Formación Profesional a la enseñanza superior. A lo largo del siglo XX ha adoptado diversos modelos y formas de llevarla a cabo, dependiendo de los agentes involucrados y los objetivos específicos. Entre ellos, el modelo de orientación entre iguales (García, 2016) ha adquirido especial relevancia al dar un lugar central al estudiante dentro del proceso de enseñanza-aprendizaje, en el marco establecido por el Espacio Europeo de Educación Superior (EEES) para la mejora de la calidad de la enseñanza. Una de las estrategias del modelo de orientación entre iguales es la de mentorización, en la que estudiantes de últimos cursos guían y supervisan el proceso de iniciación a los estudios superiores del alumnado principiante. Entre sus beneficios, fomenta el enriquecimiento mutuo y el desarrollo personal y profesional al movilizar habilidades y aprendizajes en el/la mentor/a y en el/la mentorizado/a (Skaniakos et al., 2014).

Las Escuelas Técnicas Superiores de Ingenieros (ETSI) de Telecomunicación que preparan a los futuros ingenieros e ingenieras cuentan con programas de Grado, Máster y Doctorado. Son carreras de tradición reciente que han vinculado la preparación para el ejercicio profesional con la formación científica y académica, transformándose así en opciones atractivas de altas expectativas laborales. No obstante, durante los últimos años se han detectado dificultades en el alumnado de nuevo ingreso. Esta percepción parte del estudio de los problemas a los que se enfrenta este sujeto durante el período de transición desde Bachillerato o Formación Profesional y su ingreso a la Universidad, entre los que se pueden mencionar los altos niveles de deserción y absentismo y la falta de bases teóricas para entender los contenidos de las asignaturas. En relación con el primero de los factores, los datos oficiales de algunas Universidades (Informes de Evaluación de los Títulos Oficiales de Grado) indican que la tasa de abandono inicial del título de los diferentes Grados ronda el 30\% (Universidad de Valladolid, 2016). Por su parte, la percepción del profesorado de las asignaturas respecto a los niveles de absentismo también arroja datos alarmantes. En base a una encuesta realizada al profesorado de la ETSI de Telecomunicaciones de la Universidad de Valladolid, a lo largo de los años 2017 y 2018, la mayoría evalúa la asistencia general a clases como "aceptable", "regular" o "baja/muy baja", mientras que sólo un bajo porcentaje creen que la asistencia es "buena". Las razones que aluden tienen que ver, con el alto porcentaje de estudiantes que ya han cursado esas asignaturas en años anteriores, el alto nivel de abandono que se produce en algunas asignaturas desde el comienzo del curso y el aumento del absentismo en períodos de exámenes.

Bajo este contexto surge el Programa MENTUm para detectar las dificultades personales y académicas que presenta el alumnado de nuevo ingreso en la ETSI, así como las competencias genéricas (instrumentales, interpersonales y sistémicas) que se pudieron trabajar gracias al Programa. En este artículo nos centraremos únicamente en las competencias instrumentales de aprendizaje autónomo y planificación, sin embargo, el análisis fue realizado para todas ellas en un estudio más amplio. El método empleado se enfoca en la comprensión particularista, descriptiva, heurística e inductiva de un problema (Rodríguez, 2012) con la finalidad de entender en profundidad un objeto específico, complejo y en funcionamiento.

La estructura del artículo comienza con un análisis de las experiencias de orientación entre iguales en las distintas universidades españolas. Posteriormente, se describe el programa MENTUm y la metodología de investigación realizada. Se continúa con una presentación de resultados y discusión de estos. Finalmente se presentan las conclusiones más relevantes arrojadas por dicha investigación. 


\section{Experiencias de orientación entre iguales}

Los programas de tutoría entre pares pueden ser cruciales para mejorar la experiencia de pregrado de primer año. De esta manera, los programas de tutoría de la Universidad de Melbourne (Universidad de Melbourne, 2019) permiten que los estudiantes sean guiados por estudiantes exitosos para dar consejos sobre cómo enfrentar la futura carrera. Estos programas de tutoría están disponibles en la mayoría de las áreas de grado de la universidad de Melbourne. Sin embargo, estas estrategias se vuelven cruciales en los títulos universitarios STEM (Science, Technology, Engineering and Mathematics), ya que se ha detectado una tendencia que indica una deficiencia académica en las áreas STEM para los estudiantes. Como consecuencia, los enfoques de orientación o tutoría entre pares se están integrando en universidades de todo el mundo. De esta manera, en el curso de Introducción a la Ingeniería en la Universidad de Florida Gulf Coast (Kunverger y Geiger, 2016), los estudiantes de último año actuaron como mentores de los estudiantes de primer año para aumentar su retención en los estudios de ingeniería. En este sentido, Vandana et al. (2018) presentan el diseño de un programa de tutoría entre pares para aumentar el interés en estas carreras al proporcionar interacciones entre los estudiantes en una clase introductoria de Sistemas de Información y estudiantes en un curso de Ciberseguridad de nivel superior. Zaniewski y Reinholz (2016), describen un programa de tutoría entre pares ofrecido por el Proyecto Sundial en la Universidad Estatal de Arizona para ayudar a los nuevos estudiantes para tener éxito en Física. Además, la Universidad Tecnológica de TU Delft (Universidad de TU Delft, 2019) ha diseñado el programa Estudiante por día en el que los futuros estudiantes pueden asistir durante un día lectivo a conferencias, participar en el curso de laboratorio, ver la facultad, comer en la cafetería e incluso visitar una asociación de estudiantes.

Por otro lado, las universidades españolas han dado un importante paso en el desarrollo de la orientación con una progresiva implementación de Planes de Acción Tutorial (PAT), dentro del cual destaca el modelo de orientación entre iguales y la estrategia de mentoría. Bajo este contexto, las metas y objetivos de los PAT para la educación superior se traducen en: potenciar los aspectos relacionados con el conocimiento de sí mismo/a y de los/las demás, estimular las competencias académicas y transversales que el alumnado necesita (Álvarez y Bisquerra, 2012). Estos objetivos deben desarrollarse a través de diversas herramientas y teniendo en cuenta los diferentes momentos o etapas de transición del estudiante al mundo universitario.

En la práctica, existen experiencias de mentoría entre iguales en diversas universidades españolas. La Universidad Europea de Madrid (UEM) desarrolló el programa Brújula (2006/2007), en el que estudiantes de últimos cursos con excelente expediente académico y buenas habilidades interpersonales guían a estudiantes durante su primer año universitario (Velasco y Benito, 2011). Sus objetivos se centran en mejorar el rendimiento académico y el desarrollo de competencias, así como la responsabilidad, el trabajo en equipo, la planificación y las habilidades comunicativas (Universidad Europea de Madrid, 2017). En la Universidad Pablo de Olavide (UPO) de Sevilla se realiza un proyecto dirigido a estudiantes de nuevo ingreso. Uno de los fines del proyecto es descubrir el grado de satisfacción en cuanto a la orientación académica, personal y vocacional recibida previa al acceso a la universidad y durante el primer curso. Los resultados indican que el alumnado dice sentirse bastante preparado para afrontar el reto de la universidad, pero creen que ha sido poca la influencia de la orientación recibida (Domínguez et al., 2013). Por su parte, la Escuela de Ingeniería de Telecomunicación de Vigo implementa el proyecto MEETTeleco, en el que profesorado y estudiantes de cursos avanzados actúan como mentores/as. El programa incluye una etapa de formación y otra de mentoring, donde se desarrollan reuniones entre mentores/as y alumnos/as del primer curso (Universidad de Vigo, 2017). En concordancia 
con esta experiencia de mentorización, la Universidad Politécnica de Madrid (UPM) ha implementado el Servicio de Acción Tutorial (Arroyo et al., 2012). En ese contexto, la Escuela de Informática (EUI) implementa un modelo de Tutoría por pares de tutores/as que complementa las tutorías de docentes, sumando un sistema de reuniones grupales coordinadas por alumnos/as tutores/as y varios tutelados/as. Otras experiencias, como el proyecto SOU-estuTUtor de la Facultad de Educación de la Universidad Complutense de Madrid (UCM), promueve que estudiantes de Grado interesados deben aprobar alguna asignatura vinculada al área de la orientación educativa (García et al., 2012). Por otro lado, el Programa de Alumnado en Paralelo (PAP) de la Universidad de Almería promueve la integración de estudiantes con discapacidad a través del compromiso de compañeros/as que realizan actividades de ayuda técnica o personal (Luque, 2012). La Universidad Politécnica de Valencia implementa un sistema de acogida y tutorización realizando un seguimiento más exhaustivo a estudiantes que necesiten más apoyo debido a que tienen peores resultados académicos (Balaguer, 2012; Conejero et al., 2012).

Asimismo, en la Universidad de Valladolid existen dos iniciativas respecto a la orientación universitaria, en general, y a la acción tutorial, en particular. La primera está relacionada con el Programa Orienta, que se articula en torno a una nueva concepción de tutoría universitaria, entendida como un proceso de acompañamiento continuo y de promoción del desarrollo formativo y profesional del estudiante (Cano y Paula, 2008). Durante el curso 2008/2009 se elaboró un proyecto piloto en la Diplomatura de Educación Primaria coordinado por profesores/as-tutores/as convertidos/as en agentes dinamizadores y guías del proceso. La idea era detectar aspectos académicos débiles del alumnado de nuevo ingreso y elaborar estrategias de tutoría formal y acompañamiento que permitiesen fortalecerlos, introduciendo medidas de mejora en el aprendizaje e incentivando la motivación. Este mismo Programa se llevó a cabo en la ETSI de Telecomunicación, en la Facultad de Educación y Trabajo Social, en la ETS de Arquitectura y en la Escuela Universitaria de Educación de Palencia (Gutiérrez, 2009). En este camino surge en la UVa la segunda de las iniciativas, el Programa MENTOR, un programa de mentoría grupal en el que participan alumnos/as veteranos/as (mentores/as), alumnos/as de nuevo ingreso (mentorizados/as) y docentes universitarios (coordinadores/as generales y tutores/as). Bajo ese Programa, la Escuela de Ingenierías Industriales también lo realiza con el objetivo de "ayudar a estudiantes de nuevo ingreso en su adaptación a la Universidad, favoreciendo su integración social, académica y administrativa" (Universidad de Valladolid, 2017a). Asimismo, la ETSI Agrarias de Palencia y la Escuela de Arquitectura desarrollan respectivamente, un Programa de Acción Tutorial, y un programa de mentoría entre iguales para sus estudiantes.

\section{Programa MENTUm}

En la ETSI de Telecomunicación de la UVa comenzó en 2015/2016 el Programa MENTUm, pensado como una estrategia de mentorización formal donde estudiantes de cursos superiores apoyan, supervisan y orientan al alumnado de nuevo ingreso durante todo el curso académico. Este programa está supervisado por profesores y profesoras de la Escuela y sigue la estructura jerarquizada mostrada en la Figura 1. 


\section{Figura 1.}

Estructura del Programa MENTUm

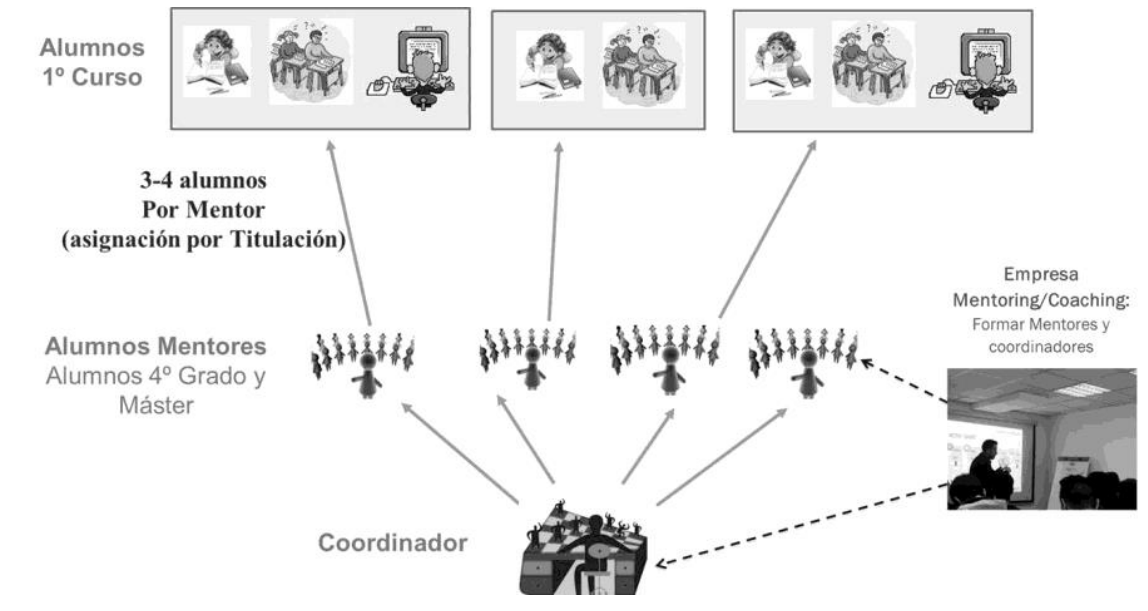

Fuente: Elaboración propia.

El objetivo principal de MENTUm es mejorar el proceso de enseñanza-aprendizaje, así como orientar e integrar al alumnado universitario de nuevo ingreso introduciendo técnicas de innovación motivadoras y atractivas. Para ello, se han establecido los siguientes objetivos específicos:

- Promover la adquisición de competencias y habilidades sociales y personales.

- Orientar el proceso de transición a la Universidad.

- Facilitar al alumnado la adquisición de competencias básicas, así como desarrollar estrategias de planificación académica.

- Proporcionar orientación académica y profesional dentro de su titulación para su futura inserción laboral.

Estos objetivos reflejan algunos de los contenidos que se pretenden desarrollar en MENTUm, sobre todo en lo que tiene que ver con el aprendizaje autónomo y la planificación y gestión del tiempo, como competencias genéricas a desarrollar en el alumnado. Para explicar el procedimiento de MENTUm, escogimos una figura cíclica (proceso circular de retroalimentación), puesto que es la que mejor se ajusta a sus características (Valverde et al., 2003).

Bajo esta estructura, la primera fase corresponde a la coordinación del Programa, en la que se evalúan las necesidades del alumnado de nuevo ingreso y se establece un plan de acción o de trabajo. La segunda fase corresponde a la formación de equipos de trabajo, que consiste en tres acciones: la selección de estudiantes que participarán como mentores y mentoras, la apertura de un período de inscripción de estudiantes de primer curso, y la formación de los grupos MENTUm (compuestos por un mentor o mentora y un máximo de tres o cuatro mentorizados/as). La tercera fase tiene que ver con la formación inicial de alumnos y alumnas mentores/as, mediante un curso de orientación en coaching dirigido por profesionales expertos. En ese curso se pretenden fomentar las competencias genéricas que MENTUm establece para ellos y ellas, tales como capacidad de liderazgo, motivación, responsabilidad social y personal, auto-orientación y elección profesional. Adicionalmente, se considera la realización de dos formaciones adicionales de seguimiento, a mediados y al final del programa.

La cuarta fase corresponde a la definición de un ciclo de acciones del proceso de mentorización y se traduce en el desarrollo de las sesiones entre mentores/as y mentorizados/as, 
programadas mensualmente. Los mentores y mentoras se encargan de programar los horarios de las reuniones con su grupo y deben cubrir temas tanto académicos como personales. Es necesario, que en algún momento de la reunión mentorizados y mentorizadas contesten un cuestionario de seguimiento con sus dispositivos móviles. Este momento está vinculado con la quinta fase, que representa la puesta en marcha del programa e involucra a todos los sujetos participantes. Durante estas dos fases se lleva a cabo tanto la ejecución del Programa como la recogida, procesamiento y análisis de los datos obtenidos a partir de los cuestionarios de seguimiento para dar paso a la última fase de evaluación y retroalimentación, destinada a evaluar tanto el impacto como la evolución de los y las participantes durante el Programa.

\section{Método}

El diseño metodológico de esta investigación está construido a partir de un estudio de caso instrumental (Simons, 2011; Stake, 2005) desde un enfoque mixto, empleando técnicas cuantitativas y cualitativas (Molina-Azorín y Fetters, 2020). Consideramos que este enfoque es el más adecuado, puesto que nuestro objetivo principal es comprender una realidad a partir del establecimiento de interrelaciones, el desarrollo de competencias y la detección de las dificultades personales y académicas que presenta el estudiantado de nuevo ingreso. MENTUm nos ayuda a detectar dichas dificultades y competencias, así como la importancia que tienen las competencias instrumentales de aprendizaje autónomo y planificación a la hora de cursar el Grado. En términos metodológicos, lo que haremos es un análisis cualitativo de los datos obtenidos, desarrollados a partir de la información que se va recogiendo (Rodríguez et al., 1999) y un análisis cuantitativo que nos sirva tanto para describir como para justificar el contexto en el cual se llevó a cabo esta investigación.

En concreto el estudio de caso nos permite entender la problemática inicial, que tiene que ver con la influencia del modelo de orientación entre iguales y la mentorización en el desarrollo de competencias genéricas instrumentales. De ahí que el programa se convierte en un instrumento gracias al cual podremos elaborar algunas respuestas en torno a las preguntas de investigación.

Para cumplir con los criterios de credibilidad, transferibilidad y confirmabilidad (Guba, 1981; Hernández et al., 2010) hemos empleado la estrategia de triangulación (Cook y Reichardt, 2005), que combina los enfoques cualitativo y cuantitativo en la recogida y análisis de datos (Creswell y Plano, 2007; Hernández, et. al., 2010) e implica la recogida de una gran variedad de evidencias, a partir de cuestionarios, observaciones y entrevistas. El diseño de investigación ha seguido las siguientes fases:

- Fase inicial: donde se presenta el programa, los objetivos, la temporalización y las condiciones de participación de los sujetos involucrados.

- Fase de implementación: se lleva a cabo la puesta en marcha de MENTUm, se realizan las observaciones, el curso de coaching y el pase de los cuestionarios inicial y de seguimiento a mentorizados y mentorizadas a lo largo del curso.

- Fase final: se hace la pasación del cuestionario final a mentores y mentoras y las entrevistas semi-estructuradas a la coordinadora del programa y a los grupos de mentores/as y mentorizados/as. 


\section{Muestra}

MENTUm es un proyecto de investigación consolidado que lleva desde el 2015 hasta la actualidad. El análisis que se presenta corresponde a los años 2017 y 2018 y cuenta con una muestra intencional (Patton, 2015) de 18 estudiantes mentores/as y 54 mentorizados/as pertenecientes a todos los Grados impartidos en la E.T.S.I.Telecomunicación de la Universidad de Valladolid. Del total de mentores/as, 5 son mujeres y 13 hombres y de mentorizados/as, 14 son mujeres y 40 hombres.

\section{Técnicas e instrumentos de recogida de datos}

Para esta investigación se utilizaron tres técnicas de recogida de datos: cuestionarios, observaciones y entrevistas. El cuestionario se utilizó para caracterizar el conjunto de participantes de MENTUm y se divide en dos etapas: la primera basada en un estudio preliminar y exploratorio y la segunda en un estudio de seguimiento a partir de las percepciones de mentores/as y mentorizados/as. Los cuestionarios se diseñaron "ah doc" para el estudio y fueron propuestos a revisión por evaluadores expertos, teniendo en cuenta los objetivos de MENTUm. Para garantizar las evidencias de validez, la adecuación y la representatividad de los ítems seleccionados procedimos a una revisión, donde los objetivos que establecimos fueron: ver la concordancia entre los evaluadores y comprobar la adecuación de los ítems para evaluar los objetivos del programa. Se utilizaron tres tipos de cuestionarios:

- Cuestionario inicial previo al comienzo de las sesiones de mentoría y dividido en:

- Ficha de datos personales y académicos

- Ficha de conocimiento sobre la titulación y el centro escogidos

- Ficha de hábitos de estudio (lugar, tiempo, estrategias, dificultades)

- Cuestionario de seguimiento al finalizar cada sesión grupal de mentoría (mensual). Se utilizó el móvil para contestar este cuestionario, lo que hacía el proceso mucho más accesible y expedito. El cuestionario tiene dos finalidades: recoger los cambios en sus hábitos de estudio, comportamiento en clase y dificultades de aprendizaje y, en segundo lugar, plantear objetivos a corto plazo (para la próxima reunión) y a largo plazo (para el fin del curso). El objetivo es evaluar de forma progresiva el proceso de enseñanza-aprendizaje del estudiantado y detectar competencias instrumentales que se estarían desarrollando y/o fortaleciendo.

- Cuestionario de fortalezas y debilidades con el objetivo de recoger las opiniones de mentores y mentoras. Este cuestionario pretende evaluar el Programa, así como el desarrollo del proceso de enseñanza-aprendizaje con sus mentorizados/as y detectar las competencias instrumentales que se estarían desarrollando y/o fortaleciendo.

El número de participantes que respondió a cada tipo de cuestionarios fue del $100 \%$. El cuestionario inicial fue contestado por los 54 mentorizados/as. Los cinco cuestionarios de seguimiento por el $92,59 \%$ de los participantes y el cuestionario de fortalezas y debilidades por el $100 \%$ de los participantes. Por otro lado, se escogió la observación para obtener información sobre el ciclo de mentoría. Dicho proceso tuvo una duración de siete meses y se compuso de 11 sesiones usando tres grupos de trabajo. Todos los datos se fueron registrando con grabaciones de audio que se transcribieron en fichas de observación, todas sobre aspectos formales y de contenido de los temas tratados.

Finalmente, se escogieron entrevistas semi-estructuradas, puesto que consideramos necesario tener un guión de preguntas que orientaran los temas a tratar con carácter flexible y ofrecer mayor libertad de expresión a los y las participantes (Verd y Lozares, 2016). Se administraron dos tipos de entrevistas en torno a dos temáticas clave: el desarrollo de 
competencias genéricas y la valoración general de MENTUm. Se realizó una entrevista con la coordinadora de MENTUm y una entrevista colectiva con los tres grupos de trabajo de las observaciones participantes. Las entrevistas fueron realizadas cara a cara, grabadas y posteriormente codificadas y analizadas por un experto en educación ajeno a la investigación, lo cual permitió contrastar la validez y la fiabilidad del estudio, así como afianzar las interpretaciones obtenidas.

\section{Resultados}

A partir de los datos recogidos, se construyeron un conjunto de categorías y subcategorías para clasificar y ordenar la información, en concreto se definieron ocho, de las cuales presentaremos los resultados de las categorías correspondientes a: Dificultades personales del alumnado de nuevo ingreso (D1); Dificultades disciplinarias o académicas del alumnado de nuevo ingreso (D2); Competencia instrumental Aprendizaje autónomo (Cl1) y Competencia instrumental Planificación $(\mathrm{Cl} 2)$. El proceso para la obtención de resultados fue la sistematización continua de las observaciones realizadas durante la inmersión inicial en el campo, estableciendo similitudes y diferencias entre las reflexiones que surgían durante el proceso de observación (Hernández et al., 2006). De este modo generamos patrones sucesivos de comportamiento que organizamos en torno a las categorías y subcategorías establecidas. Los datos obtenidos de los cuestionarios fueron analizados mediante procedimientos de análisis estadísticos para la obtención de los porcentajes de las respuestas obtenidas empleando la hoja de cálculo de Excel y los datos procedentes de las entrevistas se interpretaron siguiendo el análisis de contenido (Gil-Pascual, 2011) y las categorías establecidas permitiéndonos de este modo hacer análisis descriptivo de las opiniones de los entrevistados. Una vez finalizado el análisis de los datos de forma separada, procedemos a triangular e integrar los resultados tal y como se muestran.

\section{Dificultades personales y académicas del alumnado de nuevo ingreso}

Los resultados se centran en las categorías D1 y D2, extraídas de la fundamentación teórica en la que se apoya la implantación del EEES y que conllevó a una reformulación general del sistema educativo, reflejada en la creación de un sistema de valoración transferible del trabajo del alumnado (los créditos ECTS) y a un sistema de acreditación por competencias (Álvarez et al., 2013). No obstante, también tienen que ver con uno de los problemas transversales que atraviesan las universidades: los altos niveles de deserción y absentismo que se concentran en el primer año de ingreso. En ese sentido, González et al. (2007) indican que: "un tercio del alumnado de primer año considera seriamente la posibilidad de abandonar la educación durante ese período inicial" (p. 71). En el contexto de nuestra investigación, los índices de abandono rondan el 30\%, lo que significa un problema a nivel institucional y constituyó una de las motivaciones para la implantación de MENTUm. A partir de ese primer diagnóstico, se añadió el problema del alto grado de absentismo a clases.

Los primeros datos obtenidos, en una primera fase a través de la administración del cuestionario inicial, nos indican que los y las participantes de primer curso, tenían dificultades (personales y académicas) a la hora de enfrentarse a esta nueva etapa. Los resultados nos muestran que ante la pregunta dificultades personales que encuentras en las asignaturas, el $24,07 \%$ se refiere a problemas de concentración y el $18,51 \%$ a la falta de planificación u 
organización del tiempo. Respecto a las dificultades académicas que encuentran en las asignaturas, el $48,14 \%$ corresponde a los conocimientos de programación y falta de conocimientos previos, aspectos claramente relacionados. Lo que significa que existe relación entre deserción y absentismo, por un lado, y falta de bases teóricas o conocimientos previos, por otro, lo que va generando una sensación de inseguridad y frustración para enfrentar las asignaturas, sobre todo aquellas más complejas.

Las dificultades personales van adquiriendo importancia a medida que avanza el programa y los/las mentores/as van reconociendo una vez que se acercan de forma más profunda a sus mentorizados/as. Esto se fue viendo a través de las observaciones en las reuniones mensuales, sobre todo en momentos de más estrés y períodos de exámenes, donde se hacía más difícil planificar los tiempos de estudio o compatibilizarlos con los tiempos de ocio. Por ejemplo,

"(...) a mí me gusta muchísimo salir en plan por ahí y qué digo, ¿me quito media hora?

Pero cómo no voy a salir, es que no puedo" (Extracto de evidencia_Ficha de Observación G1, Líneas 112-120).

De ahí que sea comprensible que la mayoría de las respuestas a los cuestionarios de seguimiento relacionadas con los objetivos a cumplir estén orientadas al ámbito académico, en perjuicio del personal, puesto que la carga académica iba intensificándose progresivamente. Así, las metas que mentorizados y mentorizadas se planteaban para fin de curso tienen que ver en su mayoría (en promedio un $83,2 \%$ ) con aprobar todas o algunas asignaturas (Figura 2), mientras que los objetivos a corto plazo están relacionados con llevar al día las asignaturas o aprobar alguna de ellas (Figura 3), lo que se mantiene más o menos constante durante el periodo de investigación. Esto último está relacionado con la dificultad de planificación, puesto que a medida que avanzaba el curso se volvía cada vez más difícil organizar el tiempo para estudiar. Este problema también es manifestado por mentores y mentoras:

"No son muy objetivos con respecto a las asignaturas y piensan que tienen mucho tiempo para prepararlas" (Extracto de evidencia_Mentor 1);

"A pesar de que hacían una planificación para estudiar, les costaba empezar" (Extracto de evidencia_Mentor 2) (Cuestionario de fortalezas y debilidades).

\section{Figura 2.}

Objetivo final de curso.

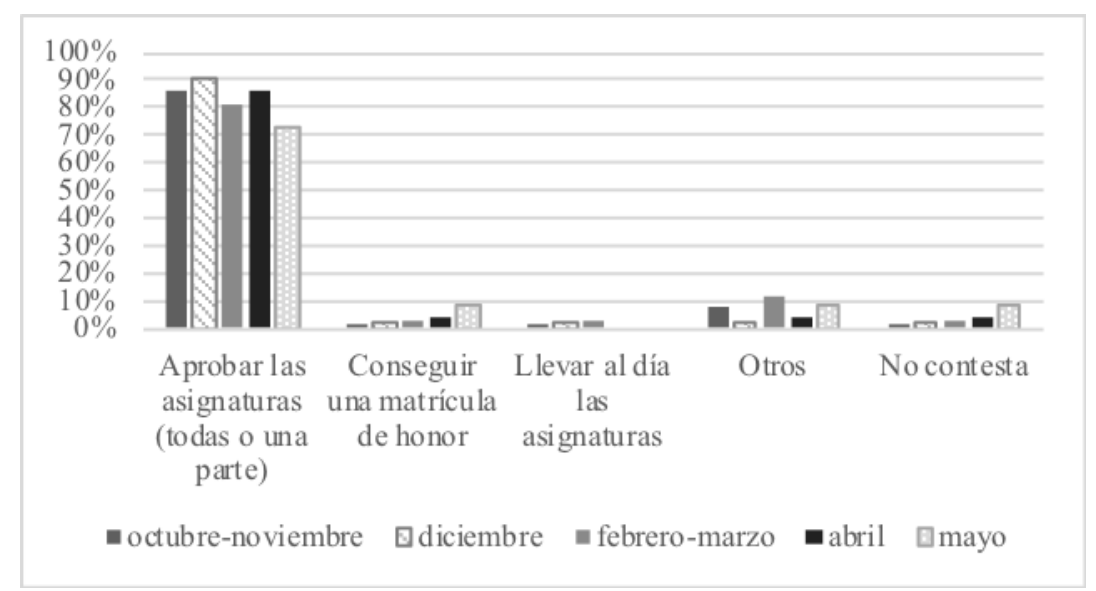

Fuente: Elaboración propia. 
Figura 3

Objetivos de la siguiente reunión.

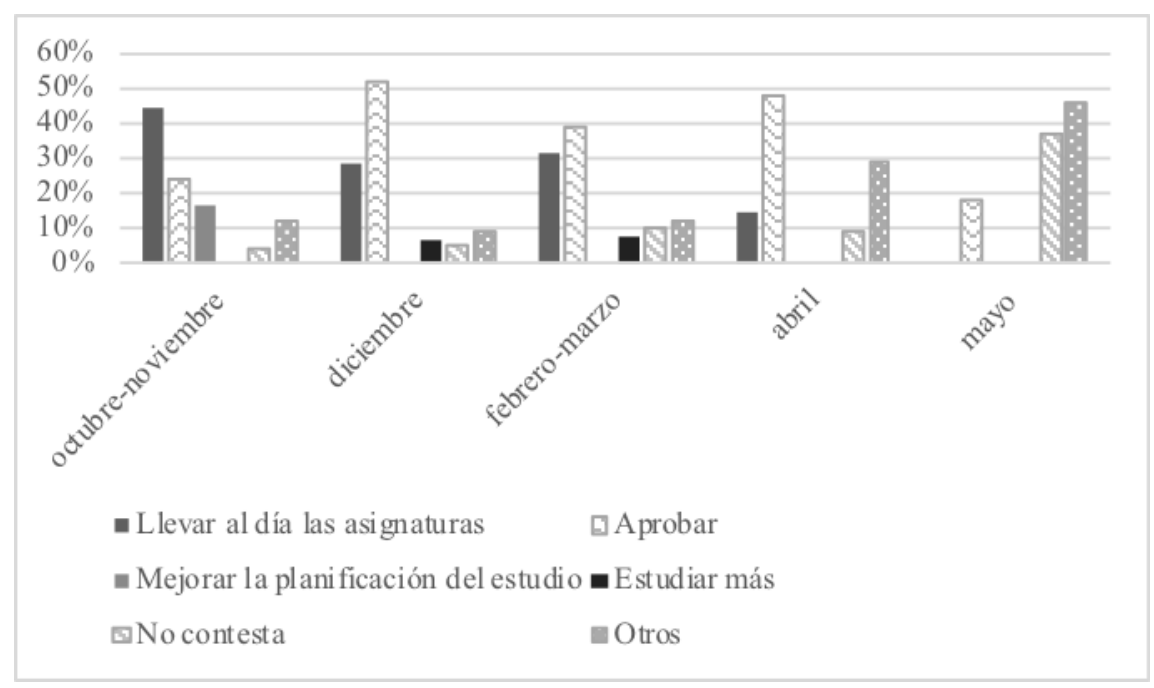

Fuente: Elaboración propia.

En función de estos resultados, es posible afirmar que las dificultades que tiene el alumnado de primer curso al ingresar a los Grados se explican, principalmente, por el cambio a nivel tanto personal como académico que se produce. Esta etapa de transición implica el cuestionamiento de las prácticas y hábitos que se mantenían en secundaria, puesto que se enfrentan a nuevas exigencias, distintos horarios de clase, otros y otras docentes y una carga académica diferente, entre otros aspectos. De ahí que una de las principales dificultades que se manifiestan sea la capacidad de planificación u organización del tiempo, sobre todo en un comienzo, puesto que se está experimentando un período de readaptación a un mundo nuevo. Todo esto, sumado a la falta de conocimientos previos, provoca una sensación generalizada de agobio, desconcentración y estrés que dificultaría compatibilizar las distintas actividades y ordenar la rutina diaria. De esta forma, se explica que un 42,58\% reconoce como principales dificultades la falta de concentración y la falta de planificación u organización del tiempo. Así pues, creemos que la identificación de las dificultades del alumnado de nuevo ingreso es clave para poder diseñar programas de orientación universitaria que se vinculen al contexto en el cual se implementan. En ese sentido, es menester hacer hincapié en esta doble dimensión de las dificultades (personales y académicas), para poder abarcar la complejidad del sujeto y adoptar un enfoque que tome en consideración su propio punto de vista para la búsqueda de estrategias y soluciones. Siguiendo a Lorenzo et al. (2014), "el estudiante, desde su ingreso en la institución universitaria, demanda ser mirado desde una perspectiva holística, integral y profunda que permita responder a exigencias que trasciendan a las meramente vinculadas con sus logros académicos" (p.32).

\section{Competencias instrumentales de aprendizaje autónomo y planificación}

En segundo lugar, analizamos las competencias genéricas, que tienen que ver con conocer y comprender (en función de los aspectos teóricos o disciplinares propios del Grado), saber cómo ser (aspectos socio-personales) y saber cómo actuar (en relación con la sociedad), para lo cual se establece la distinción entre competencias genéricas instrumentales, interpersonales y sistémicas. En esa línea, para relacionar los datos obtenidos en esta investigación con la importancia de las competencias instrumentales en estudiantes de nuevo ingreso en la ETSI hemos elaborado dos categorías que responden a las siguientes competencias instrumentales: el aprendizaje autónomo 
(Cl1) y la planificación (Cl2). Es importante recordar que esta investigación se situó en una parte de la implantación del programa y no en su totalidad, por lo que se trata de un análisis de carácter transitorio y temporal.

Los resultados de los cuestionarios administrados a estudiantes de primer año, respecto a la pregunta fortalezas personales detectadas ante cada asignatura, el 27,77\% tiene que ver con la capacidad de razonamiento y la resolución de problemas, situándose en la segunda respuesta con más frecuencia después de la capacidad de motivación (42,59\%). Esto refleja que los estudiantes son conscientes desde su ingreso a la universidad de la importancia de desarrollar estrategias de aprendizaje autónomo, que se van fortaleciendo a medida que transcurre el año académico y que van probando ciertas técnicas de estudio. Algunas de estas estrategias tienen que ver con la práctica de ejercicios en conjunto con compañeros y compañeras $y$, más importante aún, con lograr un aprendizaje de carácter eficiente. Esto se refleja sobre todo en el desarrollo de la capacidad de los estudiantes para tomar decisiones clave y enfrentarse de forma exitosa a la titulación, como puede ser el abandono de una asignatura para aprobar las demás:

"Me encontraba en Navidad y veía que Cálculo la llevaba mal y pensé dejarla e intentar aprobar las otras dos"; "Yo nunca me había planteado dejar una asignatura, al principio me costó, pero después gracias a dejarla he podido sacar bien las otras" (Extracto de evidencia_Entrevista 2, Líneas 332-334).

Por su parte, los resultados en torno a la competencia de planificación arrojan que la mayor cantidad de respuestas a la pregunta "¿En qué aspectos has evolucionado desde la última reunión?' están relacionadas con la planificación u organización del tiempo $(90,74 \%)$, en el sentido de mejorar los horarios de estudio (Figura 4), lo que refleja una mejora de esta competencia a medida que avanza el programa. Las otras respuestas que siguen en orden de frecuencia están relacionadas a esta competencia, puesto que se refieren a trabajar o estudiar de forma más constante (31,48\%) y llevar las asignaturas al día (24,07\%). Se infiere, por tanto, que la capacidad de planificación es importante para mentorizados y mentorizadas y constituye un elemento clave a lo largo del período lectivo.

\section{Figura 4}

Evolución desde la última reunión.

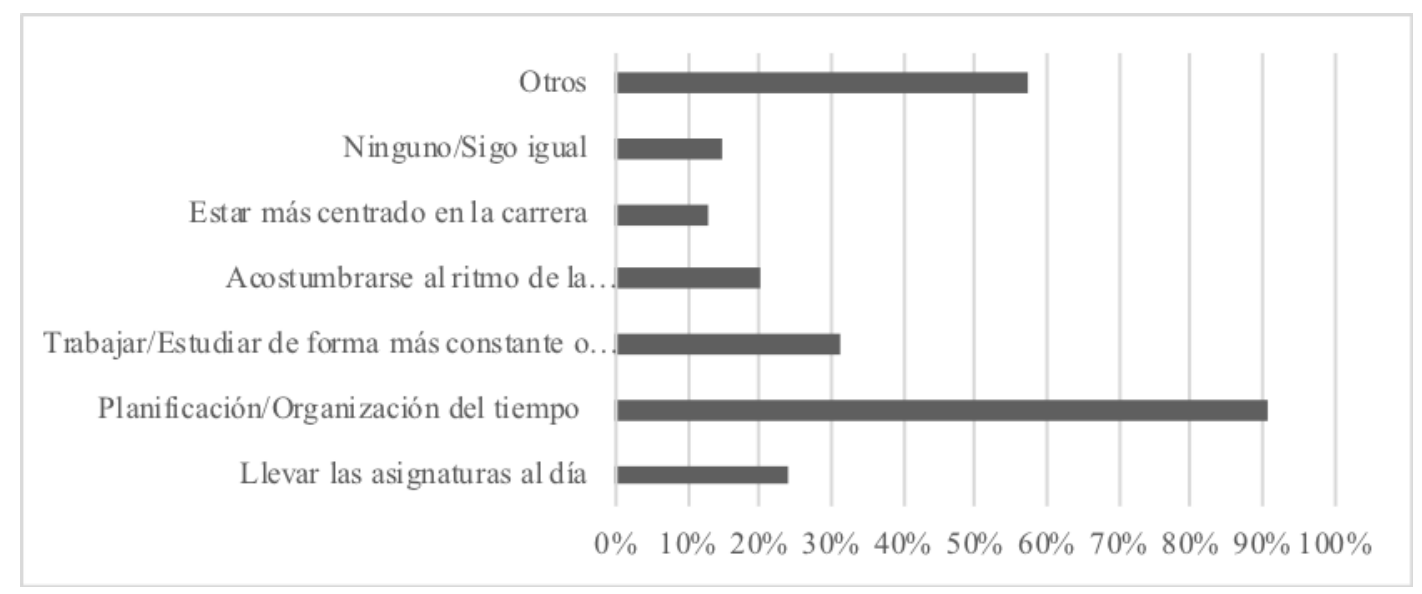

Fuente: Elaboración propia.

Finalmente, los cuestionarios de seguimiento también arrojan resultados importantes a la pregunta “¿Cómo planificas los contenidos que vas a estudiar?”, estando la gran mayoría de ellas (en promedio un 76,4\%) concentradas en la respuesta "Estudio cada asignatura en función de su examen" (Figura 5). Así, la planificación del estudio está condicionada por el calendario 
académico $\mathrm{y}$, concretamente, por el rendimiento en términos de calificaciones. Es decir, la capacidad de planificación - desde el punto de vista de mentorizados y mentorizadas - no tendría que ver necesariamente con una habilidad transversal (tal como se establece en el plan de estudios) sino que cobra relevancia en ciertos períodos de la titulación y está directamente relacionada con los resultados.

\section{Figura 5}

Planificación de contenidos.

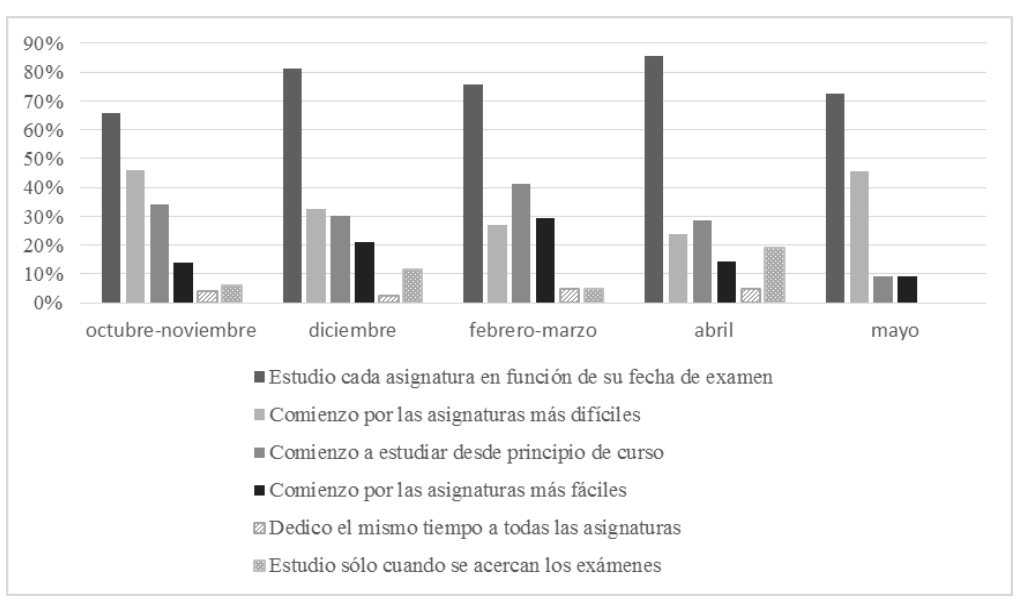

Fuente: Elaboración propia.

Estos resultados significan que el desarrollo de competencias genéricas (en este caso instrumentales) de aprendizaje autónomo es entendido como un proceso (Márquez et al., 2009) y como una medida (Solórzan, 2017), en lo referente a los objetivos, recursos y metodologías empleadas para mejorar su formación. Centrándonos en el plan de estudios del Grado en Ingeniería de Telecomunicación de la UVa, este se refiere específicamente al aprendizaje autónomo en la titulación cuando habla de la "capacidad para desarrollar metodologías y destrezas de aprendizaje autónomo eficiente para la adaptación y actualización de nuevos conocimientos y avances científicos" (Universidad de Valladolid, 2017b). De este modo, dentro de las competencias generales que establece el plan de estudios existen habilidades que fomentan el aprendizaje autónomo y que tienen que ver fundamentalmente con: la capacidad de razonamiento, análisis y síntesis; la capacidad de toma de decisiones en la resolución de problemas básicos de Ingeniería de Telecomunicación; el conocimiento de las materias básicas relacionadas con la titulación y la capacidad para comunicar. Ahora bien, a la hora de promover la autonomía en el aprendizaje intervienen múltiples y diversos factores. Se trata no sólo de incentivar el conocimiento de los contenidos básicos de la formación en la titulación, a través de hábitos de estudio, métodos de organización del tiempo u otras estrategias, sino que también de abordar situaciones complejas y encontrar soluciones óptimas y satisfactorias, como sucede en el caso de reprobaciones o mal rendimiento en algunas asignaturas. De ahí que la comunicación sea una habilidad tan relevante, así como también la tolerancia a la frustración y la comprensión empática.

En este sentido, los resultados indican que un gran porcentaje de mentorizados y mentorizadas reconoce como fortalezas personales la capacidad de motivación, el razonamiento y la resolución de problemas, que constituyen destrezas o habilidades de aprendizaje autónomo eficientes que permiten mejorar el proceso de enseñanza-aprendizaje en su conjunto. De ahí se explica que las metas tanto a corto como a largo plazo del estudiantado estén directamente relacionadas con la parte académica y las asignaturas cursadas. 
Por su parte, la categoría referida a la planificación ha sido definida como una competencia genérica de carácter transversal, que debe promoverse a lo largo de todos los cursos. En primer lugar, porque el plan de estudios del Grado hace referencia a la "capacidad de organización, planificación y gestión del tiempo" como una competencia general común a desarrollar en todas las materias, "con la finalidad de que se facilite su adquisición en cualquier entorno de desarrollo de las asignaturas del plan de estudios (...) así como en cualquier actividad formativa presencial (...) por su naturaleza básica e imprescindible" (Universidad de Valladolid, 2017b). En segundo lugar, porque los y las participantes de MENTUm le dan una especial relevancia dentro de sus intervenciones, situándola en diversas fases de su formación académica.

Uno de los aspectos en que se ve el impacto del Programa MENTUm es justamente el de la planificación. En un principio se trató de algo difícil de conseguir dentro de las reuniones de trabajo de los equipos, puesto que las dificultades previas del alumnado de primer curso tienen que ver con la organización del tiempo. Sin embargo, a medida que avanzó el programa este es uno de los aspectos más importantes que se fortalecieron durante el proceso, siendo casi la totalidad de los estudiantes quienes reconocen haber mejorado esta competencia a medida que avanzaba el programa. Se trata de una competencia de carácter transversal, a desarrollar en todos los niveles y materias, aunque cobra más relevancia en períodos de exámenes. Así, tanto el aprendizaje autónomo como la planificación son competencias que corresponden a la parte tutorial de la orientación universitaria, relacionada con el reforzamiento de habilidades de tipo instrumental.

\section{Conclusiones, Discusión y Líneas futuras}

En coherencia con los objetivos de este artículo, el Programa MENTUm permitió, en primer lugar, detectar cuáles eran las dificultades personales y académicas y la importancia de las competencias instrumentales del alumnado de nuevo ingreso de la ETSI de Telecomunicación, siendo muy significativas las dificultades personales, la falta de concentración, planificación y hábitos de estudio, coincidiendo con estudios referentes a otras titulaciones, como el de Ruiz et al. (2010) y el de Clemente y Escribá (2013). En segundo lugar, respecto a las dificultades académicas, como la falta de conocimientos previos, la base en programación y la adaptación al ritmo universitario, los resultados nos permiten concluir que se torna imprescindible reforzar la orientación académica y la nivelación de contenidos durante el proceso de transición desde la secundaria y Bachillerato, a fin de paliar el desajuste que se produce en estas asignaturas. Por último, en relación con el tercer objetivo, hay que destacar que el aprendizaje autónomo, la planificación y organización del tiempo son las competencias que más logran mejorar los y las participantes gracias a MENTUm, en concordancia con el Plan de Estudios de los Grados.

Cabe destacar la potencialidad de MENTUm por su fácil extrapolación a otros contextos educativos. Así, las fases del programa responden a un patrón genérico en este tipo de experiencias de mentoría (Valverde, et. al., 2003) y las técnicas empleadas para el análisis de las producciones podrían particularizarse fácilmente al contexto educativo pertinente, sirviendo como base para futuros proyectos de orientación universitaria. Esta experiencia de tutorización entre iguales es nueva en los Grados de Ingeniería de Telecomunicaciones y trata de incentivar el contacto entre alumnos de diferentes cursos haciendo que no solamente se cubran las necesidades académicas de los alumnos de nuevo ingreso, sino que se les apoye también desde el punto de vista personal.

Finalmente, nos gustaría indicar que la única limitación que se percibió en el programa MENTUm es que los alumnos de primer curso a medida que avanzaba el curso académico y se 
adecuaban mejor al ritmo universitario espaciaban más el contacto con sus alumnos mentores, ya que se veían más autónomos y no sentían tanta necesidad de ayuda continua de sus mentores.

Entre las líneas futuras consideramos necesario poner en marcha programas de formación previa basados en el acompañamiento, el coaching y la orientación entre iguales, ya que creemos que aporta beneficios en ambas direcciones, tanto para el alumnado mentor como para mentorizados y mentorizadas, potenciando el enriquecimiento de habilidades y competencias transversales, tal y como nos muestran los estudios de Rodríguez et. al. (2011) y Messina y Rodríguez (2006).

Para concluir nos gustaría recordar que para nosotros la mentoría significa guiar y apoyar a los estudiantes de una forma coordinada, para facilitarles las transiciones difíciles, como es el paso a la educación superior. Por tanto, este estudio nos abre a otro tipo de investigaciones como reflexionar acerca de si las buenas o malas calificaciones reflejan necesariamente el desarrollo de ciertas competencias instrumentales, tales como la capacidad de planificación, o al revés, en el sentido de preguntarse por la influencia de esta habilidad en el rendimiento académico.

\section{Referencias bibliográficas}

Álvarez, M. y Bisquerra, R. (2012). Orientación educativa. Modelos, áreas, estrategias y recursos. Wolters Kluwer.

Álvarez, P., López, D., Franchy, R., González, A. y Pérez-Jorge, D. (2013). La orientación vocacional al alumnado de bachillerato en transición a la universidad. XVI Congreso Nacional/II Internacional Modelos de Investigación Educativa de la Asociación Interuniversitaria de Investigación Pedagógica (AIDIPE), septiembre. Universidad de Alicante.

Arroyo, F., García, A., Luengo, C., Mahillo, A. y Manzano, P. (2012). Plan de Acción Tutorial en la Escuela Universitaria de Informática de la Universidad Politécnica de Madrid. Revista d'Innovació Docent Universitària, 4, 18-25. https://doi.org/10.1344/105.000001766

Balaguer, A. (2012). Análisis de la implantación de un sistema de acogida y tutorización en los nuevos estudios de grado. Simposio sobre Sistemas de Acogida y Tutorización en Estudios Universitarios (SATEU), octubre. Universidad Politécnica de Valencia.

Cano, R. y Paula, A. (2008). Programa Orienta: Plan de Acción Tutorial Universitaria para estudiantes de primer curso. Contextos Educativos, 11, 161-179. https://doi.org/0.18172/con.601

Clemente, J. S. y Escribá, C. (2013). Análisis de la percepción de las competencias genéricas adquiridas en la Universidad. Revista de Educación, 362, 535-561. https://doi.org/10.4438/1988-592X-RE-2013-362-241

Conejero, J.A., García, E. y Vivancos, V. (2012). Un modelo de tutorización y apoyo para mejorar el rendimiento académico de alumnos de 1er curso de universidad: El programa INTEGRA2. Simposio sobre Sistemas de Acogida y Tutorización en Estudios Universitarios (SATEU), octubre, Universidad Politécnica de Valencia. 
Cook, T.D. y Reichardt, CH.S. (2005). Métodos cualitativos y cuantitativos en investigación evaluativa. Morata.

Creswell, J. y Plano Clark, V. (2007). Designing and Conducting Mixed Methods Research. Sage.

Domínguez, G., Álvarez, F. J. y López, A. M. (2013). Acción tutorial y orientación en el período de transición de la educación secundaria a la universidad: La orientación al alumnado de nuevo ingreso. REDU: Revista de Docencia Universitaria, 11(2), 221-241. https://doi.org/10.4995/redu.2013.5574

García, I. (2016). Análisis de la orientación recibida por el alumnado de nuevo ingreso en la Universidad de Sevilla (Tesis Doctoral). Universidad de Sevilla.

García, M., Núñez, M., Biencinto, C. y Carpintero, E. (2012). Proyecto SOU-estuTUtor: un programa de mentoría para estudiantes de primero. Simposio sobre Sistemas de Acogida y Tutorización en Estudios Universitarios (SATEU), octubre, Universidad Politécnica de Valencia.

Gil-Pascual, J. A. (2011). Técnicas e instrumentos para la recogida de información. UNED

González, M., Álvarez, P., Cabrera, L. y Bethencourt, J. (2007). El abandono de los estudios universitarios: factores determinantes y medidas preventivas. Revista Española de Pedagogía, 236, 71-86. https://revistadepedagogia.org

Guba, E. (1981). Criterios de credibilidad en la investigación naturalista. En J. Gimeno y A. Pérez, La enseñanza: su teoría y su práctica. Akal.

Gutiérrez, J. (2009). ORIENTA: Programa de acción tutorial de la Universidad de Valladolid. Diplomatura de Educación Social. Escuela Universitaria de Educación de Palencia. II Congreso Internacional. Claves para la implicación de los estudiantes en la universidad, noviembre, Granada.

Hernández, R., Fernández, C. y Baptista, C. (2010). Metodología de la investigación. Mc Graw Hill Educación.

Kunverger T. y Geiger, C. (2016). The impact of self-efficacy in an introductory engineering course. IEEE Frontiers in Education Conference (FIE). PA: IEEE.

Lorenzo, M., Argos, J., Hernández, J. y Vera, J. (2014). El acceso y la entrada del estudiante a la universidad: Situación y propuestas de mejora facilitadoras del tránsito. Educación XXI, 17(1), 15-38. http://dx.doi.org/10.5944/educxx1.17.1.9951

Luque, A. (2012). Los sistemas de tutorización en la Universidad de Almería. Simposio sobre Sistemas de Acogida y Tutorización en Estudios Universitarios (SATEU), octubre, Universidad Politécnica de Valencia.

Márquez, A., Pascual, M. I. y Giménez, E. (2009). Desarrollo de competencias en el ámbito de los nuevos planes de estudio. VII Jornadas de Redes de Investigación en Docencia Universitaria. La calidad del proceso de enseñanza/aprendizaje universitario desde la perspectiva del cambio, junio, Universidad de Alicante.

Messina, C. y Rodríguez, A. (2006). Sentimientos, sistema de creencias y comportamiento didáctico: un estudio etnográfico. Revista de Educación, 339, 493-516. http://www.educacionyfp.gob.es/revista-de-educacion/numeros-revista-educacion/numerosanteriores/2006/re339/re339-21.html 
Molina-Azorin, J.F. y Fetters, M.D. (2020). Virtual Special Issue on Paradigms in Mixed Methods Research. Journal of Mixed Methods Research, 14 (1) 6-10. https://doi.org/10.1177/1558689819894741

Patton, M. Q. (2015). Qualitative research and evaluation methods. Sage.

Rodríguez, A., Esteban, R.M., Aranda, R.; Blanchard, M., Domínguez, C., González, C. Romero, P., Sanz, E., Mampaso, A., Vitón, M.J. y Messina, C. (2011). Coaching reflexivo entre iguales en el Practicum de la formación de maestros. Revista de Educación, 355, 355-379. https://doi.org/10.4438/1988-592X-RE-2011-355-028

Rodríguez, G., Gil, J. y García, E. (1999). Metodología de la investigación cualitativa. Aljibe.

Rodríguez, M. (2012). Cómo investigar con estudio de casos. Guía práctica. El País.

Ruiz, I., Rubia, B., Anguita, R. y Fernández, E. (2010). Formar al profesorado inicialmente en habilidades y competencias en TIC: perfiles de una experiencia colaborativa. Revista de Educación, 352, 149-178. https://dialnet.unirioja.es/servlet/articulo?codigo=3219193

Simos, H. (2011). El estudio de casos: Teoría y Práctica. Morata.

Skaniakos, T., Penttinen, L. y Lairio, M. (2014). Peer group mentoring programmes in finnish higher Education-Mentors' perspectives. Mentoring \& Tutoring: Partnership in Learning, 22(1), 74-86. https://doi.org/10.1080/13611267.2014.882609

Solórzano, Y. (2017). Aprendizaje autónomo y competencias. Dominio de las Ciencias, 1(3), 241253. http://dx.doi.org/10.23857/dc.v3i1.390

Stake, R. (2005). Investigación con estudio de casos. Morata.

Universidad de Valladolid (2016). Página web oficial de la Universidad de Valladolid. Recuperado el 18 de noviembre de 2018 de: http://www.uva.es

Universidad de Valladolid (2017a). Página web oficial de la ETSIT. Recuperado el 15 de octubre de 2019: http://www.mentor.eii.uva.es

Universidad de Valladolid (2017b). Página web oficial de la ETSIT. Recuperado el 20 de abril de 2019:de http://www.tel.uva.es

Universidad de Vigo (2017). Página web oficial de la Universidad de Vigo. Recuperado el 20 de abril de 2019 de: http://www.teleco.uvigo.es

Universidad Europea de Madrid (2017). Página web oficial de la Universidad Europea de Madrid. Recuperado el 20 de abril de 2019 de: http://www.madrid.universidadeuropea.es

University of Melbourne (2019). Official website of the University of Melbourne: Making the most of your experience. Recuperado el 20 de enero de 2020 de: https://study.unimelb.edu.au/your-experience/making-the-most-of-your-experience

University of TU Delf (2019). Official website of the University TU Delf: Student for a Day Program. Recuperado el 20 de enero de 2020 de: https://www.tudelft.nl/en/education/informationand-experience/student-for-a-day/

Valverde, A., Ruiz, C., García, E. y Romero, S. (2003). Innovación en la orientación universitaria: La mentoría como respuesta. Contextos Educativos: Revista de Educación, (6-7), 87-112. https://publicaciones.unirioja.es/ojs/index.php/contextos/article/view/530/494 
Vandana, P., Seaman, C., Kephart, K., Gangopadhyay, A. y Everhart, A. (2018). Enhancing Interest in Cybersecurity Careers: A Peer Mentoring Perspective. 49 ${ }^{\text {th }}$ ACM Technical Symposiym on Computer Science Education. Baltimore Maryland USA.

Velasco, P. y Benito, A. (2011). La mentoría entre iguales en la Universidad Europea de Madrid: Una estrategia educativa para el desarrollo de competencias generales y específicas. Higher Learning Research Communications, 1(1), 10-32. https://doi.org/10.18870/hlrc.v1i1.32

Verd J. y Lozares, C. (2016). Introducción a la investigación cualitativa. Fases, métodos y técnicas. Síntesis.

Zaniewski A. y Reinholz D. (2016). Increasing STEM success: a near-peer mentoring program in the physical sciences. International Journal of STEM Education, 3(14), 1-9. https://doi.org/10.1186/s40594-016-0043-2

Fecha de entrada: 31 marzo 2020

Fecha de revisión: 28 mayo 2020

Fecha de aceptación: 8 septiembre 2020 